\title{
3-D Pain Drawings-Mobile Data Collection Using a PDA
}

\author{
Gheorghita Ghinea, Member, IEEE, Fotis Spyridonis, Tacha Serif, and Andrew O. Frank
}

\begin{abstract}
A large number of the adult population suffers from some kind of back pain during their lifetime. Part of the process of diagnosing and treating such back pain is for a clinician to collect information as to the type and location of the pain that is being suffered. Traditional approaches to gathering and visualizing this pain data have relied on simple 2-D representations of the human body, where different types of sensation are recorded with various monochrome symbols. Although patients have been shown to prefer such drawings to traditional questionnaires, these pain drawings can be limited in their ability to accurately record pain. The work described in this paper proposes an alternative that uses a 3-D representation of the human body, which can be marked in color to visualize and record the pain data. This study has shown that the new approach is a promising development in this area of medical practice and has been positively received by patients and clinicians alike.
\end{abstract}

Index Terms-3-D visualization, mobile data collection, pain drawings.

\section{INTRODUCTION}

I T IS estimated that $80 \%$ of the adult population suffer from back pain during their lifetime. Besides being uncomfortable and affecting day-to-day life on a personal level, it has a considerable effect on society and the economy [1], [2]. In spite of this, the assessment of this medical complaint remains notoriously difficult.

One of the first steps that is traditionally undertaken by patients in a back pain clinic is the completion of a medical questionnaire, which is intended to identify the location and type of pain being experienced.

In most cases, the only visual aid to assist medical staff with their assessment is "pain drawings." Traditional pain drawings are 2-D figures of the human body (Fig. 1) on which the patient is asked to mark the type and distribution of the pain being suffered. The pain drawings, in conjunction with a psychological evaluation, allow the physician to assess whether the pain is anatomic or nonanatomic. Moreover, studies have indicated that patient drawings have high completion rates in a study comparing patients completing conventional questionnaires and pain drawings [3]. Notwithstanding their advantages, 2-D pain drawings have their limitations, as they do not capture the 3-D nature

Manuscript received February 9, 2006; revised April 27, 2007.

G. Ghinea, F. Spyridonis, and T. Serif are with the School of Information Systems, Computing and Mathematics, Brunel University, UB8 3PH Uxbridge, U.K. (e-mail: george.ghinea@brunel.ac.uk; fotis.spyridonis@brunel.ac.uk; tacha.serif@brunel.ac.uk).

A. O. Frank is with the Rheumatology Department, Northwick Park Hospital, HA1 3 Harrow, U.K. (e-mail: andrew.frank1@btinternet.com).

Color versions of one or more of the figures in this paper are available online at http://ieeexplore.ieee.org.

Digital Object Identifier 10.1109/TITB.2007.903266

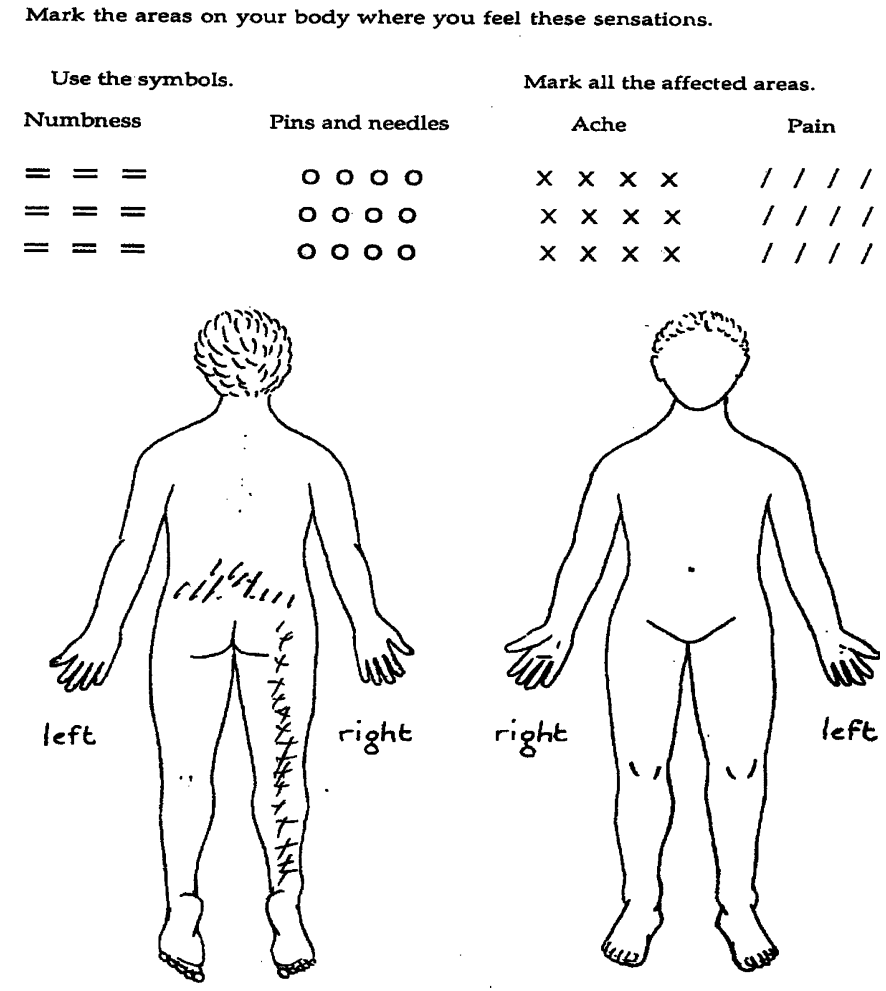

Fig. 1. Completed 2-D pain drawing.

of the human body. Thus, patients are unable to visually express the pain that they are experiencing, as statements of the form "I have a pain on the inside of my thigh" are not easily captured in a 2-D pain drawing.

While recent inventions of medical imaging techniques such as computerized tomography and magnetic resonance imaging have revolutionized radiology, the development of 3-D imaging has not yet been ported across the world of pain drawings. The focus of the work described in this paper has been the augmentation of the traditional 2-D pain drawing with a novel, computer-based, 3-D version--in the anticipation that electronic 3-D pain drawings shall increase the ease with which patients record their own pain, as well as providing a tool for pain data collection and monitoring for back pain clinicians. Moreover, we have ported the developed 3-D pain drawing to a personal digital assistant (PDA) platform, and implemented a wireless solution for mobile data collection. Accordingly, the structure of this paper is as follows. Section II provides a more detailed overview of back pain visualization, while Section III introduces the ideas behind the 3-D pain drawing concept. Section IV describes the design of a PDA-based 3-D pain drawing prototype for wireless data collection, which is then evaluated in Section V. 
Lastly, conclusions are drawn and opportunities for future work identified in Section VI.

\section{BACK PAIN VISUALIZATION}

The diagnosis and treatment of low back disorders (LBDs), and specifically, of back pain is a major health problem that research in medical as well as biodynamic areas is currently dealing with. According to past data, $60-80 \%$ of the population will suffer some form of back pain during their life, with a considerable negative impact on the health budgets and national economies of countries [1], [4].

Unfortunately, the available medical information provides only partial success in the diagnosis and treatment of this chronic disease with only $15 \%$ of the patients obtaining an accurate diagnosis of their problem [3]. This partial success is due to the complexity of the back, where the main causes of pain could result from reasons such as strains and minor injury rather than serious injury, originating from wrong sort of movement to muscle and spine abnormalities. In addition, back pain can also be triggered by injury to back muscles or spinal discs during lifting with improper load handling [1], [5].

Like most types of pain, back pain is difficult to assess, as the only information that can be used is suggestive descriptions from the patient. However, because these patients may have developed psychological and emotional problems as a result of suffering with pain, it may be difficult for them to provide a clinician with wholly accurate information. This, in turn, can lead to problems with treatment. This problem is further exacerbated as, in some patients, the psychological problems may have actually caused some of the back pain by adding stress to the body, or the stress of the back pain may have caused psychological problems [6].

\section{A. Pain Drawings}

One of the key developments in back pain diagnosis was developed by Ransford et al. [7]. Their technique involved the use of body outlines where patients were asked to mark on the type (usually ache, pain, pins and needles, and numbness) and distribution of the pain being experienced. These diagrams were called "pain drawings."

Previous research [8] had indicated that the hypo-chondriasis (Hs) and hysteria (Hy) scores of the Minnesota Multiphasic Personality Inventory (MMPI) were the best prognosticators for disc-disease. The Ransford et al. study showed that pain drawings were a good indicator of Hs and Hy scores, and thus, could effectively be used in the treatment and management of back pain.

Over the years, there have been a number of research studies to continue and extend research in respect to pain drawings. Accordingly, there have been modified adaptations of the initial pain drawing system and the scoring system. All of the studies [5], [9]-[11] had slight variations but were conceptually the same. They shared the same diagrammatic framework and used mathematical analysis for pain scoring. In most cases, there was an outline of the human body, with one view from the front and another from behind. Originally without any divisions, in later studies, each such diagram had regional selections already marked on, however still using only two outlines. It is also noticeable that all these approaches tend to share the same or similar notations to indicate the different types of pain being experienced (aches, numbness, pins and needles, burning, and stabbing). Confirming earlier results [7], most of these subsequent developments proved to be good indicators of back pain and accurately distinguished between physiological and psychological causes [5], [6], [12].

Masferrer et al. [11] further explored the use of color as a representation for the different types of pain being experienced. The results from this study showed that the ability of color pain drawings to express the pain experienced remained the same as with previous approaches. However, upon analysis of the drawings, physicians found it easier to see distinctions between colored representation of pain rather than previous monochrome notations.

\section{3-D PAIN DRAWINGS}

Previous sections have highlighted that back pain information is mainly gathered through the use of interactive interviews by highly skilled medical personnel, supported by various paperbased questionnaires. However, the 2-D representation of pain drawings constitute a limited dimension representation of the medical information, potentially resulting in a time-consuming process with possible irrelevant medical data collected that can lead to a report that obscures important information. Therefore, it seems natural to implement digitized 3-D pain drawings, since such an approach provides an attractive opportunity for enhancing interaction between the practitioner and the patient in a more perceivable way to the natural environment. Nonetheless, in our research, it was felt important that technology should not be applied to the problem for the sake of it, but that any new approach should be more intuitive than existing approaches, but just as usable.

\section{A. Need for 3-D}

In computer technology, the concept of 3-D is intimately associated with that of virtual reality. This is the simulation of a real or imagined environment that can be experienced visually in the 3-D of width, height, and depth, and that may additionally provide an interactive experience, usually by manipulating keys or the mouse so that the content of the image moves in some direction or zooms in or out. By being able to interact with the environment, anomalies caused by 2-D depth perception can be removed, potentially allowing for more accurate and consistent data collection.

In the case of pain visualization, it was felt, after consultations with clinicians and patients alike, that a patient would be able to better map the pain that they were experiencing onto a 3-D model of themselves rather than a 2-D one. Using a front and rear 2-D projection of a human torso makes it difficult to map pain that is occurring at the edges of the representation. A 3-D model provides a continuous body surface, which allows a practitioner, or indeed, a patient, to accurately record the pain. In the past, it was often considered to be expensive for day-to-day 
activity. However, developments have made it a standard tool for utilization.

In terms of back pain, an experimental study using 3-D for reconstructing spinal cord injuries reinforced the power of 3-D in medical practice. 3-D virtual images were constructed from performing computerized medical scans [1]. In this case, 3-D images were extremely beneficial because the models could be observed from many different viewpoints. Rotation and zooming features were combined to allow observer navigation within the tissue. 3-D navigation provided depth perception, so spatial relationships of features could be better revealed. The same feature benefit was anticipated from devising a 3-D adaptation of pain drawings.

\section{B. Need for a Mobile Solution}

Because of the complexity and sensitivity of back pain problems, every sufferer needs individual treatment options. However, unavoidable situations such as queues at hospitals or practitioners' individual places of treatment, which are prioritized based on the level of emergency or sickness, cause type 2 patients (abdominal pain, chronic back pain, etc.), who are not considered as urgent cases, to have to wait excessively long, in order for their health-related information to be collected. Moreover, assuming that individual treatment and prescription has been given, it is natural that the patients want to be informed regularly of their health situations, and the doctors on their part to be able to monitor their patients' medical situation, therefore, to have both easy and relatively fast access to such health information.

Sadly enough, conditions such as the hospital queues mentioned before, among others of course (since this is only a very simple example to demonstrate the situation), do not allow an effective and efficient use of health information that would provide both parts with the necessary means for disorder, specifically, back pain disorder in our case, diagnosis, and treatment. The rapid acquisition and distribution of such information is definitely a priority, but most of the times, the professionals responsible for such activities often operate under tight time constraints. Therefore, health-related information collection and allocation in an effective and systematic way must be balanced with the fact that they need to attend to all patients as promptly as possible [13].

Because of the enormous impact of the aforementioned conditions, there have been efforts [9], [10], [14] to guide the patient's diagnosis, treatment, and monitoring outside clinics in a way that health-related issues can be efficiently manipulated and used in a timely manner, since time and space constitute barriers between health care providers and their patients, and indeed, among health care providers themselves. One of these directions has been to empower the patient to become a stakeholder in the management of pain. Moreover, bearing in mind the need for an anywhere, anytime connection for medical information access, a more flexible and mobile telemedicine system could be developed that would be able to overcome the current health-related information limitations discussed so far. This is precisely what we address in this paper, and describe our experiences with the design of a PDA-based solution for mobile data collection of 3-D pain data.

\section{PRototype DESign}

\section{A. Requirements}

The design of the prototype was conducted in collaboration with a team of clinicians from the Rheumatology Department of the Northwick Park Hospital, London, who would potentially use the system. Interviews with these stakeholders were held in order to define and refine the desired requirements of the prototype. In particular, interviews focused on the deficiencies of the existing back-pain assessment approach and areas of opportunity for the 3-D tool to possibly exploit. In brief, the identified requirements were to:

1) provide a 3-D model of the human body;

2) provide fully navigational controls enabling the ability to zoom, rotate, drag for depth perception;

3) allow individually selectable regions of the body;

4) use color to represent different types of pain;

5) allow the details (pain location, type, intensity, time of input) to be saved for later analysis by physician and for record keeping on a patient's file;

6) provide the patient with the ability to input back-pain data ubiquitously and upload it to a central server;

7) provide a handheld solution for data collection, since many back pain patients, due to associated disabilities, are confined to wheelchairs or beds, and would not have easy access to desktop systems.

\section{B. Delivery Platform}

The developed 3-D back pain application was designed and implemented, based on previous work of ours [14], by combining the efficiency of Microsoft embedded Visual Basic, a language specifically geared to help developers build applications for the next generation of communication-and-informationaccess devices running Windows $\mathrm{CE}$, and the functionality of the Cortona SDK, a development component that enables Virtual Reality Modeling Language (VRML) browsing.

Using the VRML component, our application displays a 3-D human mannequin (Fig. 2) whose surface was segmented into clinically appropriate regions after consultations with the medical staff involved in our research. Based on earlier work [5], [15], we color coded four different types of pain (numbness, pain, pins and needles, and ache). Pain intensity is also inputted for a particular region of the body via a visual analog scale (implemented in our prototype via a scroll bar).

The user has zoom-in and zoom-out buttons for manipulating the mannequin (Fig. 3), whereas rotations are implemented through user stylus input. In order to input the type of pain that $\mathrm{s} /$ he is currently experiencing, the user employs the stylus to first select the pain type, after which $\mathrm{s} /$ he manipulates the mannequin to the desired level of detail, and with the help of the touch-sensitive screen, indicates the particular region of the body that is affected. This is then recorded to a local Microsoft Pocket Access database file. It is notable to mention that even 


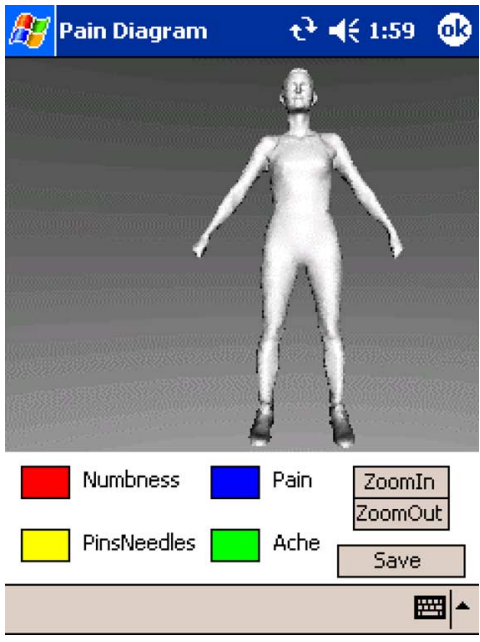

Fig. 2. PDA-based 3-D pain visualization interface.

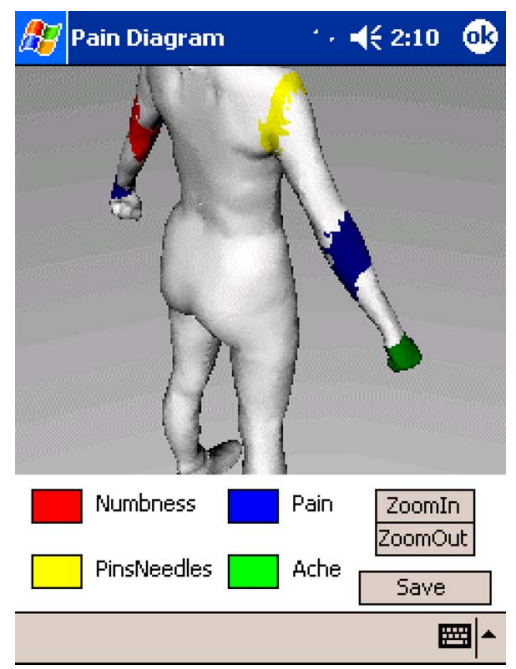

Fig. 3. Rotated and zoomed-in 3-D pain drawing, highlighting areas of pain.

though data input is mainly done on the 3-D mannequin, the data that are saved are not pictorial, but numerical (location of pain--each body region had a unique identifier, type of pain, intensity of pain, and time of day). As such, database sizes are relatively small [14] and can be uploaded using standard dialup connection speeds (under $45 \mathrm{~s}$ using a $56-\mathrm{kb} / \mathrm{s}$ modem to transfer a typical day's worth of collected data).

Data can be uploaded either through the standard synchronization tools via the PDA cradle, or when the user is within wireless Internet coverage. In both cases, the application uses Winsock CE 3.0 (Windows CE Sockets) to send its database content to a central server, which runs Windows 2003 Server operating system and Internet Information Server (IIS) 6.0 with an Open Database Connectivity (ODBC) service.

The system architecture in Fig. 4 shows the main components that make the 3-D wireless back-pain recording model work. Accordingly, the 3-D Back-Pain Application was implemented on an HP iPAQ 5450 PDA with 16-b touch-sensitive transflective thin-film translator (TFT) liquid-crystal display (LCD) that supports 65536 color. The display pixel pitch of the device is

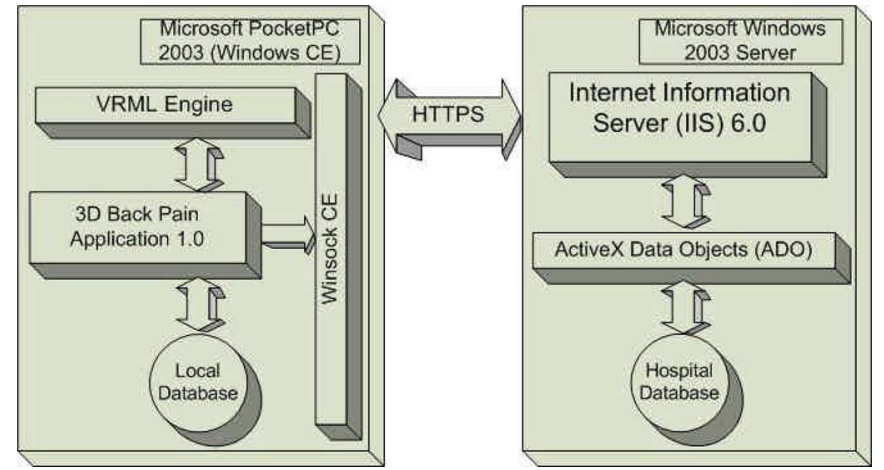

Fig. 4. System architecture for delivery of a wireless 3-D pain visualization solution.

$0.24 \mathrm{~mm}$, and its viewable image size is 2.26 in wide and 3.02 in tall. It runs Microsoft Windows for Pocket PC 2003 (Windows CE) operating system on an Intel $400-\mathrm{MHz}$ XScale processor and contains $64 \mathrm{MB}$ standard memory as well as 48-MB internal flash ROM. The Web server was implemented on an Intel Pentium IV running at $3.4 \mathrm{GHz}$, with 1-GB RAM and a 250-GB hard disk. Throughout our trials, a Netgear DG834PN 108-Mb/s RangeMax ADSL Modem Wireless Router was used.

Due to the sensitivity of the data sent from the patient's handheld device, all the information is sent through WiFi Protected Access-Pre-Shared Key (WPA-PSK) encrypted radio broadcast at wireless level. At software level, the information privacy is maintained by the use of 128-b Secure HyperText Transfer Protocol (HTTPS). In addition to all of the above, to prevent identity theft, rotating-password approach is utilized, which requests five random characters of predefined 16-letter password, and creates a unique keyword combination for every transfer.

\section{Evaluation}

The purpose of the initial evaluation of the prototype was twofold: first, to gain feedback from the clinicians involved in developing the requirements, and second, to gain an understanding of how usable the interface was by potential patients.

\section{A. Clinical Evaluation}

Four clinicians were asked to review the prototype. One was a back-pain specialist from a London hospital, another was a palliative care specialist from a different London hospital, with the prototype also being evaluated by two physiotherapists. This review was to ascertain whether this approach to pain visualization could be used in practice, by professionals with considerable experience in the use of existing 2-D pain drawings.

In general, all clinicians surveyed approved of the visual appearance of the system, and suggested that the prototype would be usable in a clinical environment. They provided a number of interesting observations on limitations and improvements that could be made.

The back pain specialist noted that the 3-D interface covered almost all aspects of existing pain drawings. He was impressed by the level of detail and navigation control. However, he did note that users with disabilities might find it difficult to interact 
with the PDA. While he was "excited" by the possibility of patients collecting their own data, especially at set times of the day (and thus, being able to remotely monitor the progression and type of pain, vis-a-vis the prescribed medication/treatment), he did highlight that 1) users should be given appropriate training and 2) appropriate personnel and facilities should be made available to interpret this wealth of data, otherwise it would ultimately be a futile exercise. These concerns were also echoed by the palliative-care clinician, who was, however, impressed by the opportunity that the application gave patients to become stakeholders in managing their pain. Moreover, he was also of the opinion that even though the tool did not provide a diagnosis as such, it could have important and benefic psychological effects on patients eager to record their pain diaries.

Both physiotherapists interviewed had no concerns with the usability of the prototype, but did suggest that 1) the feet should point downward rather than be in a standing position to allow for ease of marking and analysis and 2) we could, in future versions of the prototype, consider having a more fine-grained division of the mannequin body surface. The physiotherapists were impressed by the potential ability of the prototype for anytime, anywhere data collection, and hinted that even if the prototype would have had usability issues, in practice, most patients would overlook this, as the convenience factor associated with it would outweigh such considerations--for one, there would be less hospital visits!

\section{B. Patient Evaluation}

Forty-five patients (26 males, 19 females, mean age 46.1 years) have evaluated the prototype between September 2006 and January 2007. Out of these, 13 were patients at the Rheumatology Clinic of Northwick Park Hospital and volunteered to take part in the study. The remaining 32 participants were members of the U.K. National Forum of Wheelchair User Groups.

The only qualifying condition was that participants had to have broadband access at home and allow us to install the wireless router over the five-day evaluation period (although wireless connectivity is not necessary for pain data upload, it was considered essential in order to evaluate this aspect of our prototype). Patients were given a three-page user manual and asked to record pain data at three set times a day, subsequently uploading the information at the end of each day to the hospital server. At the end of the evaluation period, patients were asked to complete a questionnaire (Table I) in which their opinions over a range of statements were recorded on a 5-point Likert scale, where 5 corresponded to the most positive response and 1 to the most negative. Users were also asked to note any other comments they might have had in respect of the developed application.

In general, the results were positive. Users found the color notation clear, and found it easy to navigate and control the 3-D model used in the PDA application (Fig. 5). These results with respect to the developed interface are especially encouraging, since the majority of the users were wheelchair patients, many of whom wore glasses, and whose condition was compounded by other disabilities (such as motor ones).
TABLE I

Questions UsEd to EVAluATE THE 3-D PDA PAIN Visualization TOOL

\begin{tabular}{|l|c|c|}
\hline \multicolumn{1}{|c|}{ Question } & $\begin{array}{c}\text { Mean } \\
\text { Response }\end{array}$ & $\begin{array}{c}\text { Standard } \\
\text { Deviation }\end{array}$ \\
\hline $\begin{array}{l}\text { Q1. How effective did you find } \\
\text { the controls to navigate the } \\
\text { model? }\end{array}$ & 4.24 & 0.83 \\
\hline $\begin{array}{l}\text { Q2. How would you describe } \\
\text { the overall layout of the }\end{array}$ & & \\
interface? & 4.31 & 1.00 \\
\hline Q3. Were the tool tips helpful? & 4.29 & 0.89 \\
\hline $\begin{array}{l}\text { Q4. Was the use of the color } \\
\text { notation clear? }\end{array}$ & 4.29 & 0.94 \\
\hline $\begin{array}{l}\text { Q5. It is important to be able to } \\
\text { record my pain on a PDA. }\end{array}$ & 4.38 & 0.86 \\
\hline $\begin{array}{l}\text { Q6. It is useful to be able to log } \\
\text { pain data across time. }\end{array}$ & 4.40 & 0.78 \\
\hline $\begin{array}{l}\text { Q7. It was difficult to input pain } \\
\text { data on a PDA }\end{array}$ & 2.07 & 0.75 \\
\hline $\begin{array}{l}\text { Q8. Process of transferring data } \\
\text { from PDA to the main database } \\
\text { could be easier }\end{array}$ & 1.98 & 0.72 \\
\hline
\end{tabular}

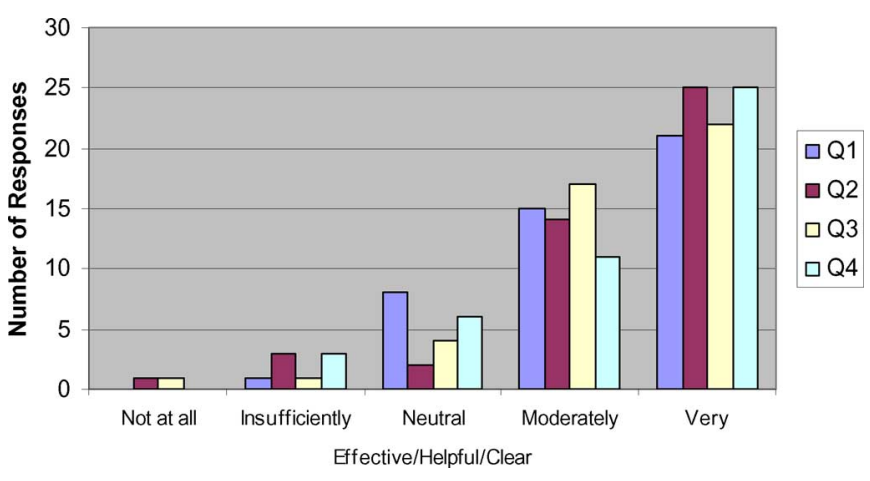

Fig. 5. Breakdown of survey responses: questions 1-4.

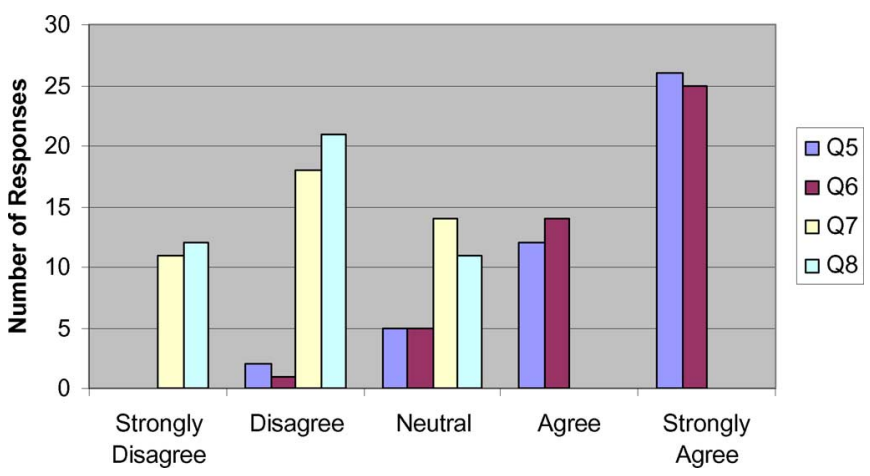

Fig. 6. Breakdown of survey responses: questions 5-8.

Strongly positive results were obtained with respect to the ability of the prototype to record pain data anywhere, anytime, and especially, with the fact that it allows the patient to show clinicians how their pain varies across the day, in this respect, the trend confirming earlier results of ours [14]. Furthermore, patients generally disagreed with statements regarding the difficult of data input and upload (Fig. 6).

Some of the comments made by patients when evaluating the application include: 
"The application allows me to correlate more closely the pain I am experiencing with the activities that I had been doing ..."

The patient then remarked that, as a result of self-monitoring of how activities impacted his/her pain levels, he/she could manage the pain much better by reducing those activities that led to intense pain.

Another patient remarked:

"The software made me realize that I was taking my medication at the wrong time of the day ..."

This observed discrepancy between medication intake and experienced peaks of pain, as well as a better understanding of the link between activities undertaken during the day and pain intensities and patterns in the end resulted in the patient reducing his medication (strong analgesic) by $25 \%$, with no deterioration in the pain levels encountered. Indeed, the reduction of medication intake as a result of self-monitoring of pain was not a singular observation, as this was reported by five other members of our study group.

Yet another patient highlighted:

"Being able to rotate, zoom-in and out makes me feel that I have a much better control of my pain ..."

This observation seems to show that even though pain levels of the individual in question had not necessarily decreased as a result of the developed application, at least it offered him a perceived leverage of control over his suffering. Indeed, as remarked earlier, surveyed users were most enthusiastic about the ability to $\log$ data and its variation in time.

In rounding off our analysis of participants' comments, we mention that we were particularly pleased with the statement to the effect that:

“... 3-D on a PDA allows me to accurately pinpoint any location I choose. Now, I would never go back to 2-D (pain drawings) again!"

Lastly, we remark that, from the additional written and oral comments that we have received, the general trend was that patients were enthusiastic about the application, with the only suggestion for further improvements being a finer division of the body mannequin, so that pain locations could be better pinpointed and the possibility of having more than one type of pain for each selected body region.

\section{CONCLUSION}

This paper has presented a novel, PDA-based application for ubiquitous upload of pain data gathered using a 3-D pain drawing. Our evaluation with a cohort of 45 users has revealed encouraging results, with patients appreciating the advantages that self-monitoring of pain offers in managing this complex phenomenon, while clinicians especially liked the ability of the developed application to remotely monitor pain. Moreover, both patients and clinicians indicated positive views toward the developed interface, platform, and functionality.

We realize that the cost (of equipment and software) is a potential issue toward large-scale adoption of our work. However, we are heartened by two aspects in this respect: the prices of PDAs are decreasing, while their computational capacity is increasing, as is their connectivity. Moreover, any potential initial investment in equipment and software could be offset by the reduction in health care costs (better pacing of medication intake, as our study has highlighted; potentially fewer hospital visits) as well as by increased patient satisfaction due to the opportunity to become a stakeholder in the management of pain (again, as shown by our study, although we realize that it is difficult to put a price on patient satisfaction).

The work reported in this paper has raised interesting future directions, chief of which is the use of color to code multiple pain types for a particular body region. While our proof-ofconcept prototype has not taken into consideration issues such as application-level security and scalability; clearly, these are avenues for future work, as are more fine-grained subdivisions of the 3-D mannequin. The application could also be ported to other clinical areas (such as patients recovering from surgery or being treated for cancer). Our research is, of course, prototypical. It has not been tested in a large-scale setting, nor has its impact on health care processes been assessed. All these efforts constitute an essential part of our future endeavors.

\section{REFERENCES}

[1] A. O. Frank and L. H. De Souza, "Conservative management of low back pain,” Int. J. Clin. Pract., vol. 55, no. 1, pp. 21-23, 2001.

[2] P. M. Wolsko, D. M. Eisenberg, R. B. Davis, R. Kessler, and R. S. Phillips, "Patterns and perceptions of care for treatment of back and neck pain: Results of a national survey," Spine, vol. 28, no. 3, pp. 292-297, 2003.

[3] S. Duerstock, C. L. Bajaj, V. Pascucci, D. Schikore, K. N. Lin, and R. B. Borgens, "Advances in three-dimensional reconstruction of the experimental spinal cord injury," Comput.Med.Imag. Graph., vol. 24, no. 6, pp. 389-406, 2000.

[4] M. L. Vaughn, S. J. Cavill, S. J. Taylor, M. A. Foy, and A. J. B. Fogg, "Using direct explanations to validate a multi-layer perceptron network that classifies low back pain patients," in Proc. 6th IEEE Int. Conf. Neural Inf. Process., vol. 2, pp. 692-699.

[5] H. Parker, P. L. Wood, and C. J. Main, "The use of the pain drawing as a screening measure to predict psychological distress in chronic low back pain," Spine, vol. 20, no. 2, pp. 236-243, 1995.

[6] A. Uden, M. Astrom, and H. Bergenudd, "Pain drawings in chronic back pain," Spine, vol. 13, no. 4, pp. 389-392, 1988.

[7] A. O. Ransford, D. Cairns, and V. Mooney, "The pain drawing as an aid to psychologic evaluation of patients with low-back pain," Spine, vol. 1, no. 2, pp. 127-134, 1976.

[8] L. L. Wiltse and P. D. Rans, "Preoperative psychological tests as predictors of success of chemonucleolysis in the treatment of the low-back syndrome," J. Bone Joint Surg., vol. 57, no. 4, pp. 478-483, 1975.

[9] E. J. Gómez, C. Cáceres, D. López, and F. Del Pozo, "A web-based selfmonitoring system for people living with HIV/AIDS," Comput. Methods Programs Biomed., vol. 69, no. 1, pp. 75-86, 2002.

[10] C.-H. Lin, S.-T. Young, and T.-S. Kuo, "A remote data access architecture for home-monitoring health-care applications," Med. Eng. Phys., vol. 29, no. 2, pp. 199-204, Apr. 2006.

[11] R. Masferrer, V. Prendergast, and P. Hagell, "Colored pain drawings: Preliminary observations in neurosurgical practice," Eur. J. Pain, vol. 7, no. 3, pp. 213-217, 2002.

[12] D. Ohnmeiss, "Repeatability of pain drawings in a low back pain population," Spine, vol. 25, no. 8, pp. 980-988, 2000.

[13] J. R. Warren, D. E. Warren, and R. W. Freedman, “A knowledge-based patient data acquisition system for primary care medicine," in Proc. 2nd Int. Conf. Infor. Knowledge Manage., Washington, DC, Nov. 1993, pp. 547553.

[14] T. Serif and G. Ghinea, "Recording of time-varying back-pain data: A wireless solution," IEEE Trans. Inf. Technol. Biomed., vol. 9, no. 3, pp. 447-458, Sep. 2005.

[15] K. M. Prkachin, I. Schultz, J. Berkowitz, E. Hughes, and D. Hunt, "Assessing pain behaviour of low-back pain patients in real time: Concurrent validity and examiner sensitivity," Behav. Res. Therapy, vol. 40, no. 5, pp. 595-607, 2002. 


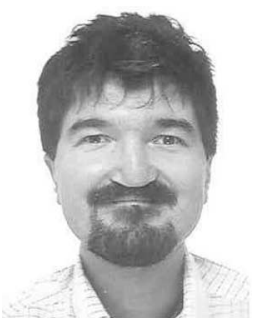

Gheorghita Ghinea (M'02) received the B.Sc. and B.Sc. (Hons.) degrees in computer science and mathematics, and the M.Sc. degree in computer science from the University of the Witwatersrand, Johannesburg, South Africa, in 1993, 1994, and 1996, respectively, and the Ph.D. degree in computer science from the University of Reading, Reading, U.K., in 2000.

$\mathrm{He}$ is a Reader in the School of Information Systems, Computing and Mathematics, Brunel University, Uxbridge, U.K. His current research interests include pervasive computing, telemedicine, quality of service, and multimedia resource allocation, as well as computer networking and security issues.

Fotis Spyridonis received the M.Sc. degree in distributed information systems and computing in 2006 from Brunel University, Uxbridge, U.K., where he is currently working toward the Ph.D. degree in the School of Information Systems, Computing and Mathematics.

His current research interests include telemedicine and pervasive computing.

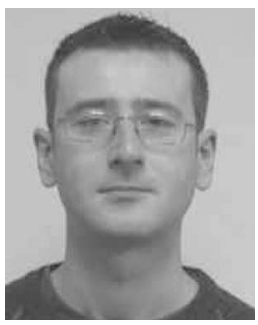

aware systems.

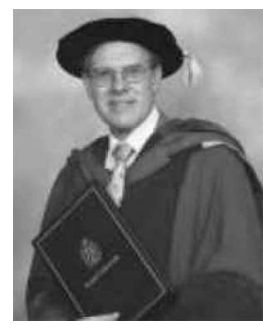

Andrew Oliver Frank received the M.B.B.S. degree from the University of London, London, U.K., in 1968, the M.R.C.P. and F.R.C.P. degrees from the Royal College of Physicians of London, London, in 1971 and 1990, respectively, the D.Sc. (Hons.) degree from Brunel University, Uxbridge, U.K., in 2003, and the F.H.E.A. degree from the Higher Education Academy, York, U.K., in 2005.

$\mathrm{He}$ is currently with the Rheumatology Department, Northwick Park Hospital, Harrow, U.K., where he was appointed as a Consultant in 1980. He has also been a Consultant to the Stanmore Specialist Wheelchair Service in 1997 and Harrow PCT's Physical Disability Support Team in 1999. He was also an Associate Professor in the Department of Health Sciences and Social Care, Brunel University, in 1997. He is the author or coauthor of different books on disabling diseases and low back pain.

Mr. Frank edited the British Society of Rehabilitation Medicine (BSRM) reports on Vocational Rehabilitation in 2000 and 2003 and was its President from 2000 to 2002. He is also the Founder Chair of its Vocational Rehabilitation Special Interest Group. He was the Clinical Chair of the National Health Service (NHS) Modernization Agency Wheelchair Services Collaborative and chaired its faculty during 2002-2004. He was the recipient of an honorary D.Sc. from Brunel University in 2003. He is also a Trustee of the Vocational Rehabilitation Association. He chairs the Professional Advisory Board of Kynixa, Ltd. 